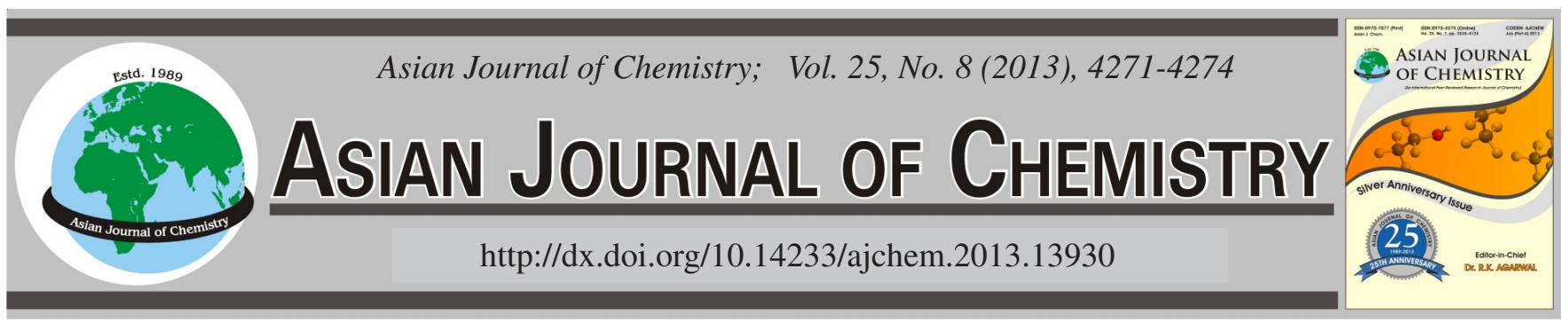

\title{
Macroporous Resin Column Simulated Moving Bed Chromatography Adsorption for Separation of Albiflorin and Paeoniflorin
}

\author{
Wei ZhANG ${ }^{*}$, QiAN Li and SHAOYAN WANG
}

University of Science and Technology, Liaoning, Anshan 114044, Liaoning Province, P.R. China

*Corresponding author: Fax: +86 412 5929480; Tel: +86 412 5929030; E-mail: askdzw@163.com

\begin{abstract}
D101 macroporous resin column simulated moving bed separation process of albiflorin and paeoniflorin isomers was applicated in the paper. Determine the simulated moving bed separation conditions of albiflorin and paeoniflorin, separation column for the D101 macroporous resin column (ID. $26 \mathrm{~mm} \times 400 \mathrm{~mm}$ ), simulated moving bed chromatographic operation mode :1-4-3, sample concentration: $250 \mathrm{mg} / \mathrm{mL}$, sampling flow rate: $=2 \mathrm{~mL} / \mathrm{min}$, elution rate: $=20 \mathrm{~mL} / \mathrm{min}$, extraction flow rate: $=35 \mathrm{~mL} / \mathrm{min}$, switching time: $=25 \mathrm{~min}$, elution and extract: methanol: water $(\mathrm{v} / \mathrm{v})=20: 80$. Calculated according to the standard curve, mass concentration of paeoniflorin in product was $90 \%$, the yield was $78 \%$; albiflorin was $91 \%$, the yield was $75 \%$. Under these conditions, the system can be stable operation. The relevant factors that impact of simulated moving bed separation was also discussed in the paper.
\end{abstract}

Key Words: Fine separation, Simulated moving bed chromatography, Macroporous resin, Albiflorin, Paeoniflorin.

\section{INTRODUCTION}

Peony is Paeonia paeonia Lactiflora Pall.'s dry root without skin, has anti-inflammatory, anti-virus, solution spasm and pain, protect the liver and other pharmacological activities ${ }^{1}$. Paeoniflorin is the main active ingredient, belongs to monoterpene glycosides, paeoniflorin has lower blood sugar ${ }^{2}$, cardiovascular protection $^{3}$, regulation of the immune system ${ }^{4}$, anti-tumor effects ${ }^{5}$. Albiflorin is also the active ingredient, also belongs to monoterpenoid glycosides ${ }^{6}$, albiflorin has weak inhibition of DNA split ${ }^{7}$ and the anticonvulsant effects ${ }^{8}$. In strctures, paeoniflorin and albiflorin ${ }^{9}$ both have the same molecular formula, same molecular weight, different structures are the typical isomers.

Simulated moving bed chromatography (SMBC) is a new development of a large-scale chromatographic separation technology ${ }^{10}$, it is combination of the simulated moving bed technology and chromatography technologies. It retains the advantages of fine separation by the chromatographic, turn the chemical technology as continuous, counter current, rectification, reflux and other factors intensive in, has a large-scale fine separation ability ${ }^{11,12}$.

In recent years, study on the albiflorin and paeoniflorin' pharmacology were more extensive, so demand for the albiflorin and paeoniflorin monomer were increasing. Traditional extracts of total glucosides of peony is a mixture containing both ${ }^{13}$, now more commonly be used separation technology for albiflorin and paeoniflorin is column chromatography, that not only causes a large number of waste in solvent, while limits precision of separation ${ }^{14-16}$.

China scholars have studied simulated moving bed in separation of traditional Chinese medicine intermediates, technology that separation of peony extract by the simulated moving bed not only improving the content of the isolates to a new height and open a simulated moving bed technology applications new areas. Simulated moving bed chromatography with macroporous resin column separation albiflorin and paeoniflorin not only improve the purity of the product, while increasing the yield of the product, saving the solvent to achieve a low-carbon production of environmental conservation. By application of high-capacity simulated moving bed we achieved high-precision separation of albiflorin and paeoniflorin in total glucosides of peony, separation products were determined by the content of $90 \%$ or more.

\section{EXPERIMENTAL}

Extracts of total glucosides of peony (Shaanxi days of the Biotechnology Co. Ltd.); paeoniflorin standards and albiflorin standards (national standard center); macroporous resin column (ID. $26 \mathrm{~mm} \times 400 \mathrm{~mm}$, Section on Biological Development Co. Ltd.); D101 macroporous resin (Resin Technology Co. Ltd. Anhui Samsung); reagents were analytical grade reagents. 
WAD-1 refrigerated air desiccant: Hangzhou Lin'an Wanda Electrical Equipment Manufacturing Co. Ltd., Automatic control of simulated moving bed system (Liaoning University of Technology made), simulated moving bed chromatography column 8 with the preparation of (ID. $26 \mathrm{~mm} \times 400$ $\mathrm{mm}$, Beijing welcomes the development by the Bioscience Limited) and the injection pump: Beijing Star Technology Co. Ltd. (the largest flow $10 \mathrm{~mL}$ min), elution pump: Beijing Star Technology Co. Ltd. (maximum flow $30 \mathrm{~mL}$ min), extraction pumps: Beijing Star Technology Co. Ltd. (maximum flow $50 \mathrm{~mL}$ min). Simulated moving bed chromatography running mode: 1-4-3, sample concentration: $100 \mathrm{mg} / \mathrm{mL}-300 \mathrm{mg} / \mathrm{mL}$, injection flow rate: $=2 \mathrm{~mL} / \mathrm{min}$, elution rate: $=10-25 \mathrm{~mL} / \mathrm{min}$, extraction flow rate: $=25-50 \mathrm{~mL} / \mathrm{min}$. Switching time: $=15-60 \mathrm{~min}$ and the extraction liquid eluent:methanol:water $(\mathrm{v} / \mathrm{v})=20: 80$.

Shimadzu LC-10 AT liquid chromatograph, SPD-10A UV detector, Agilent column (ODS packing, $5 \mu \mathrm{m}, 4.6 \times 150 \mathrm{~mm}$ ), flow rate: $=1.0 \mathrm{~mL} / \mathrm{min}, \lambda=230 \mathrm{~nm}$, mobile phase: methanol: water $(\mathrm{v} / \mathrm{v})=30: 70$, injection volume: $20 \mu \mathrm{L}$.

Drawing standard curve: The precise amount of a certain amount of albiflorin and paeoniflorin standards placed in $50 \mathrm{~mL}$ volumetric flask, using methanol to volume, dubbed the concentration was $0.54 \mathrm{mg} / \mathrm{mL}$ of standard mixed solution, precise amounted of 1.0, 3.0, 5.0, 7.0, 10.0 mL standard mixed solution in $10 \mathrm{~mL}$ volumetric flask with methanol to volume, dubbed a series of concentration of the solution. Injected in the analysis conditions, analyzed by the HPLC. The reference standard peak area for the vertical coordinate $(\mathrm{Y})$, the concentration for abscissa (X), draw the standard curve. The results showed that paeoniflorin concentration in the range of $0.054-0.54 \mathrm{mg} /$ $\mathrm{mL}$ as a good linearity, the regression equation was $\mathrm{Y}=$ $207.8808 \mathrm{X}+4.9801, \mathrm{r}=0.9994$; albiflorin concentration in the ranger of $0.054-0.54 \mathrm{mg} / \mathrm{mL}$ ae a good linear, the regression equation was $\mathrm{Y}=108.7906 \mathrm{X}+3.9925, \mathrm{r}=0.9997$.

Preparation of simulated moving bed raw material: The total glucosides of peony extract dissolved with a certain amount of ethanol, filtered insoluble, freeze-drying the solution to collect dry matter content. Preparated of a certain concentration of total glucosides of peony raw material solution with pure methanol by 1.2 require and set aside. HPLC analysis graph was shown in Fig. 1.

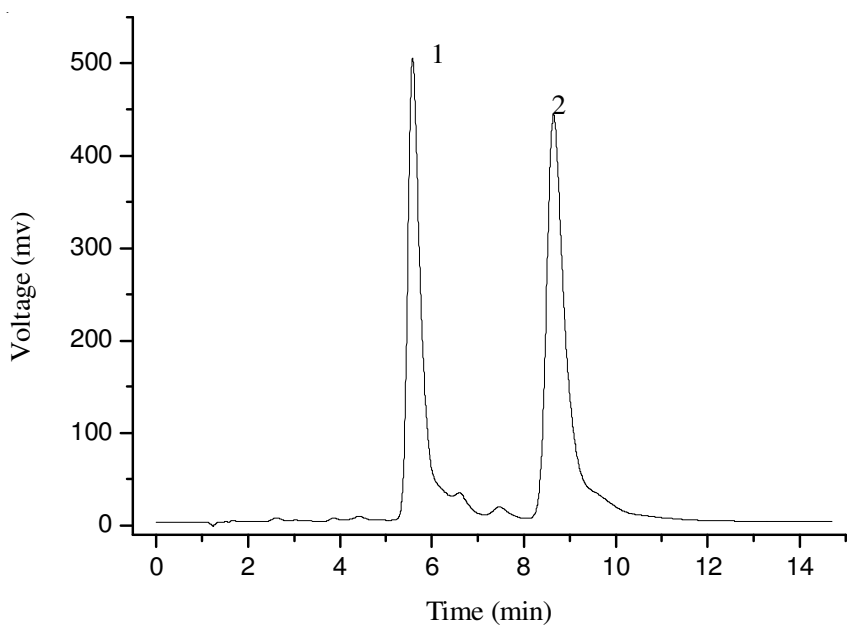

Fig. 1. Chromatography of raw material for simulated moving bed (1. albiflorin; 2. paeoniflorin)
Separation of albiflorin and paeoniflorin isomers by simulated moving bed chromatography: Simulated moving bed materials were dubbed the sample solution, simulated moving bed separation of albiflorin and paeoniflorin isomers was experimented in preparation conditions. Albiflorin as the fast component out from the raffinate port (R port), paeoniflorin as the slow component out from the extraction port (E Port). As the simulated moving bed is a nonlinear multi-degree of freedom separation system, separation results of the product (mass fraction and the yield) depend on the optimization and choice of the system parameters, experiment carried out in accordance with the conditions described in regulation 1.2, separation results of the product as evaluation index, adjusted and optimized the separation conditions. HPLC analysis of the albiflorin and paeoniflorin isomers separated by simulated moving bed were shown in Fig. 2.
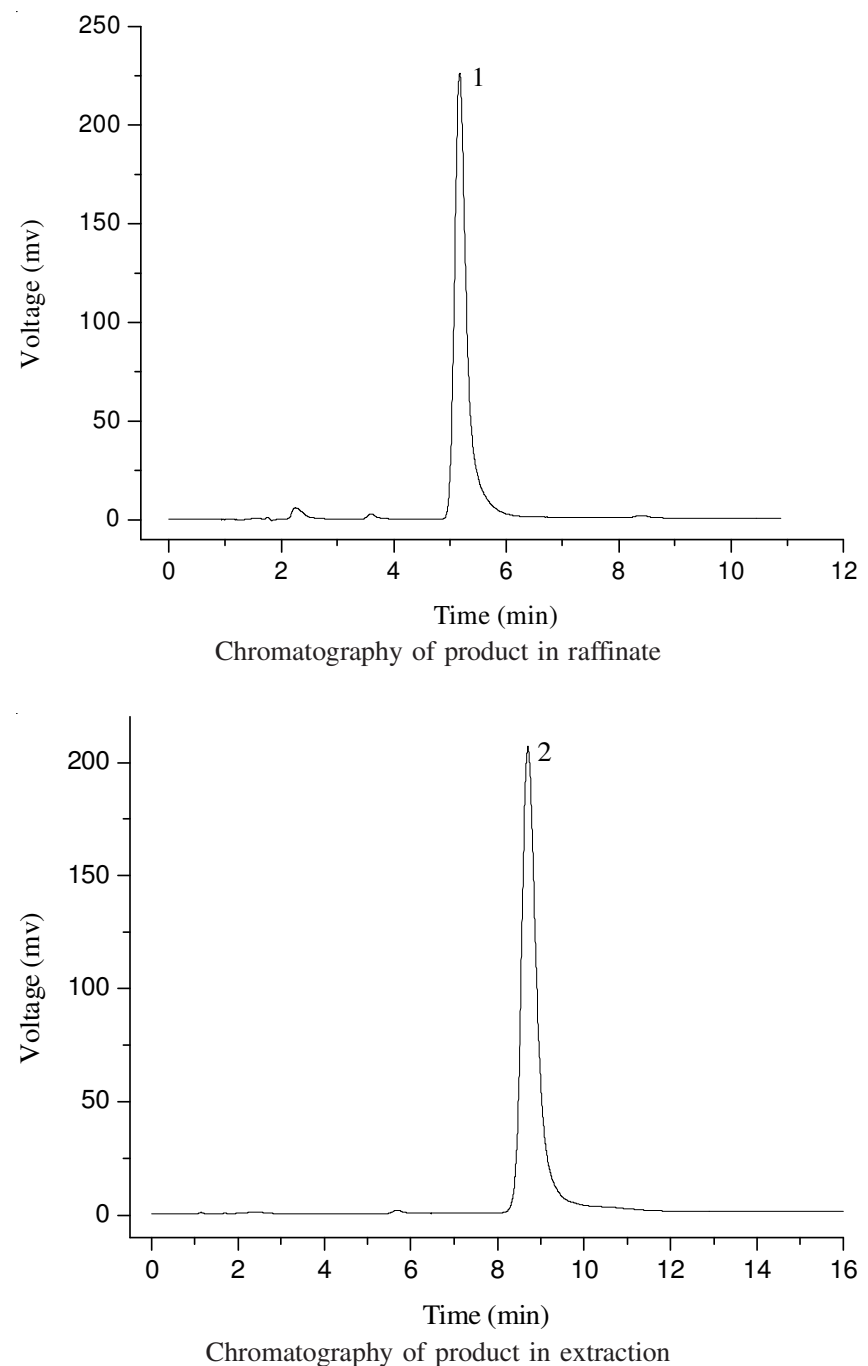

Fig. 2. Chromatography of product in raffinate and extraction of simulated moving bed (1. albiflorin; 2. paeoniflorin)

\section{RESULTS AND DISCUSSION}

Choice and influence of rejection concentration: On Simulated moving bed separation experiments, the concentration of raw materials solution and product mass concentration and yield have a direct relationship. According to the relationship between the mass concentration of albiflorin and 
paeoniflorin in sample solution and the yield of albiflorin and paeoniflorin in the product mixture made concentration curve (Fig. 3).

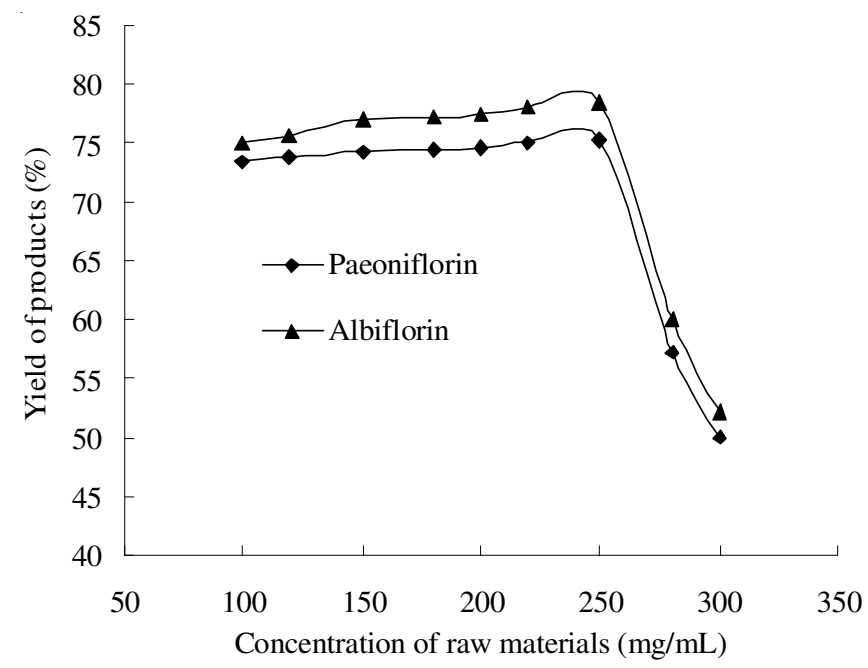

Fig. 3. Curve of the injection mass concentration on yield of product

Fig. 3 shows the injection concentration changes directly affected the product yield, when the injection concentration was $250 \mathrm{mg} / \mathrm{mL}$, yield of albiflorin and paeoniflorin in the product were highest scores while the mass concentrations met experiments requirements. Therefore we chosen $250 \mathrm{mg} /$ $\mathrm{mL}$ as injection concentration.

As the simulated moving bed technology is a scale fine separation technology, in order to obtain greater production, we need to increase the injection volume of raw materials and we usually increase injection concentration to achieve increasing injection volume, the increase of injection concentration is bound to make preparation volume has non-linear effects. Therefore, most of the simulated moving bed separations are in non-linear conditions, for the nonlinear effects of two components, we amendment by Morbidelli triangle ${ }^{12,17}$. In this study, the concentrations of albiflorin and paeoniflorin were in the non-linear range of the resin colume adsorption, resulted in the chromatographic curves of albiflorin tailing and chromatographic curve of paeoniflorin protrusion. In order to achieve the balance of maximum yield and maximum rejection volume, including the rejection concentration for the experiment, a series of simulated moving bed operation parameters were determined by a number of trials and ultimately determine the range of the best separable concentration.

Choice and influence of elution rate: In the experiment, we draw the curve of plate height and the flow rate (Fig. 4). That shown that when the flow rate was $20 \mathrm{~mL} / \mathrm{min}$, the plate height minimum, then preparation columns had the highest column efficiency, so the choice of elution flow rate was 20 $\mathrm{mL} / \mathrm{min}$.

In simulated moving-bed experiments, elution rate is an important factor, the flow rate is too large, not only result in waste solvents and sometimes can affect the quality of products; flow rate is too small, leading to prolonged simulated moving bed switch time, reducing the separation efficiency. In addition, according to the equation of the plate height and the flow rate (Van-Deemter equation), will also affect the separation effect of column, thereby affecting the separation. Fig. 4 shows that, compare Van-Deemter curve, when the flow rate near the 20 $\mathrm{mL} / \mathrm{min}$, plate height was minimum, column efficiency was the highest, separation efficiency was the strongest, when the flow rate was greater than $20 \mathrm{~mL} / \mathrm{min}$, the plate height was increased with increasing flow rate, column efficiency was reductied, separation also reduced the mass concentration of the product. If the preparation column is smaller, when determinating of Van-Deemter curve, minimum point of plate height is not easy to measure, then the optimum flow rate should be determined based on specific experimental results.

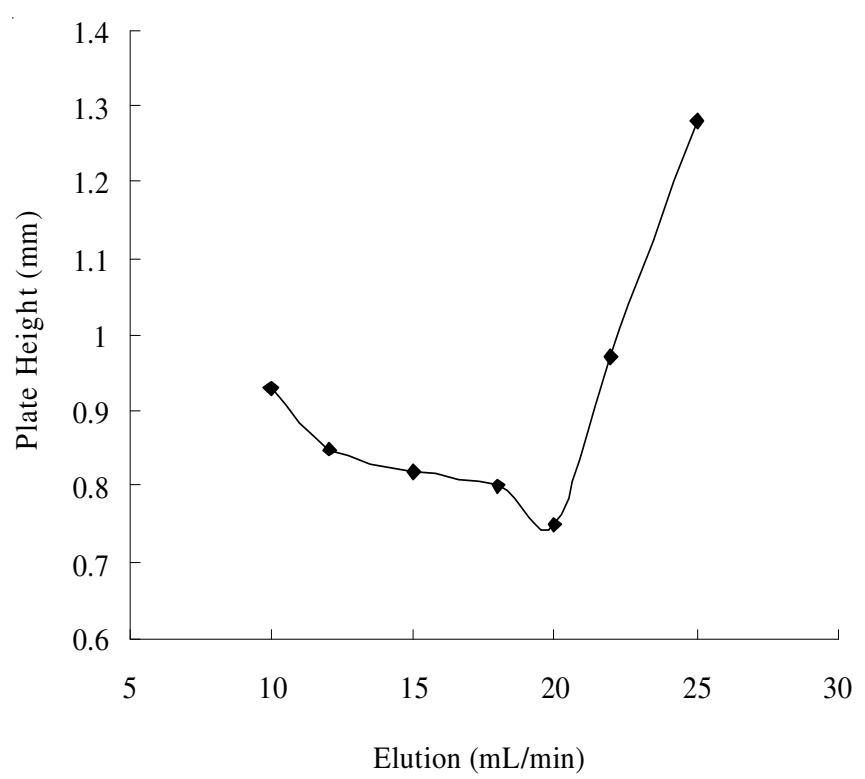

Fig. 4. The Van-Deemter graph of elution rate and plate height

Choice of switching time: Switching time is one of the simulated moving bed important operation parameters, based on the fractions analysis structure of albiflorin and paeoniflorin in a resin column, we drawed curve of albiflorin and paeoniflorin peak height changes with time, shown in Fig. 5.

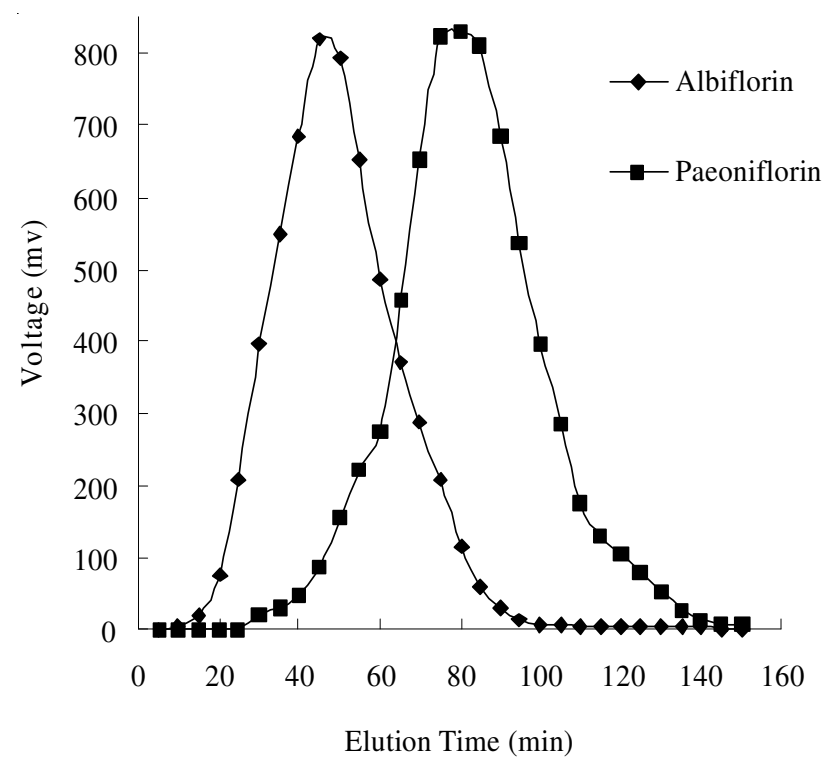

Fig. 5. Curve of peak and elution time 
According to Fig. 5, we know that albiflorin increased speed got larger at $25 \mathrm{~min}$, albiflorin yield and quality percentage are more appropriate at $40 \mathrm{~min}$, as the result of paeoniflorin at $80 \mathrm{~min}$ is also ideal, but switching time is too long, albiflorin product mixed with paeoniflorin, the yield and quality of paeoniflorin reduced. Therefore, the switching time was chosen to be $25 \mathrm{~min}$. Fig. 5 shows at the switching time of $25 \mathrm{~min}$, in a switching point albiflorin and paeoniflorin concentration distribution in the 8 resin columns.

Fig. 6 shows that albiflorin and paeoniflorin in a simulated moving bed chromatography system achieved a better separation.

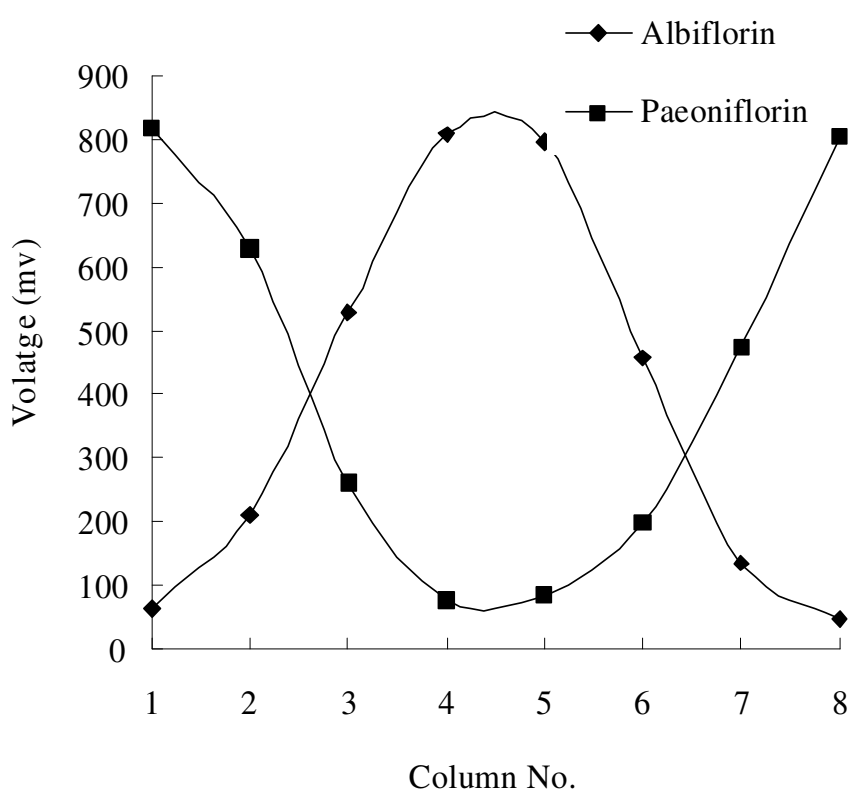

Fig. 6. Concentration distribution curve of albiflorin and paeoniflorin in 8 resin columns

Simulated moving bed operation parameters: After simulated moving-bed operating parameters were adjusted several times to finalize the D101 macroporous resin column simulated moving bed operating parameters for the separation conditions of albiflorin and paeoniflorin isomers: separation column for the D101 macroporous resin column (ID $26 \mathrm{~mm} \times$ $400 \mathrm{~mm}$ ), simulated moving bed chromatography operation mode :1-4-3, sample concentration: $250 \mathrm{mg} / \mathrm{mL}$, injection flow rate: $=2 \mathrm{~mL} / \mathrm{min}$, elution rate: $=20 \mathrm{~mL} / \mathrm{min}$, extraction flow rate: $=35 \mathrm{~mL} / \mathrm{min}$, switching time: $=25 \mathrm{~min}$, elution and extract: methanol: water $(V / V)=20: 80$. Separated under these conditions, Calculated according to the standard curve, mass concentration of paeoniflorin in product was $90 \%$, the yield was $78 \%$; albiflorin was $91 \%$, the yield was $75 \%$. Achieved separation albiflorin and paeoniflorin isomers by simulated moving bed. Continuously operated the system 100 cycles did not find major changes for product quality, proved that could be stably operated.

\section{Conclusion}

Experiments with D101 macroporous resin column simulated moving bed equipment successfully separated the albiflorin and paeoniflorin isomers, mass concentration of paeoniflorin in product was $90 \%$, the yield was $78 \%$; albiflorin was $91 \%$, the yield was $75 \%$ and the yield was high. Simulated moving bed equipment is simple and when the injection concentration was $250 \mathrm{mg} / \mathrm{mL}$, the system could continuously and stably operatd, product handling capacity was big, while saving solvents. Experimental results show that, components of Chinese medicine raw materials are complex and running state of simulated moving bed separation substances must be required in the nonlinear concentration, so the best simulated moving bed running experiments require a combination of operating parameters to be adjusted.

\section{REFERENCES}

1. X.-Y. Zhang, J.-H. Wang and X. Li, J. Shenyang Pharm. Univ., 18, 30 (2001).

2. S.-F. Lu and Y.-J. Shi, Planta Med., 63, 323 (1997).

3. R. Sun, L.-L. Lv, S.-D. Guo and G.-Q. Liu, et al., J. Harbin Univ. Comm., 21, 406 (2005).

4. Z.-D. Ge, A.-W. Zhou and B. Wang, Chinese Pharmacol. Bull., 11, 303 (1995).

5. X.-S. Yan, H.-M. Li, Y.-Q. Peng and A.-P. Ming, Chinese J. Integrat. Trad. Western Med. Liver Diseases, 16, 18 (2006).

6. M. Kaneda, Y. Iitaka and S. Shibata, Tetrahedron, 28, 4309 (1972).

7. T. Okubo, F. Nagai, T. Seto, K. Satoh, K. Ushiyama and I. Kano, Chem. Pharm. Bull., 23, 199 (2000).

8. A. Sugaya, T. Suzuki, E. Sugaya, N. Yuyama, K. Yasuda and T. Tsuda, J. Ethnopharm., 33, 159 (1991).

9. Q. Wang, R.X. Liu, H.Z. Guo, M. Ye, C.H. Huo, K.S. Bi and D. Guo, Chromatographia, 62, 581 (2005).

10. B.-C. Lin, Fine Chem., 22, 110 (2005).

11. W. Zhang and B.-C. Lin, Fine Chem., 24, 554 (2007).

12. G. Paredes, M. Mazzotti, J. Stadler, S. Makart and M. Morbidelli, Adsorption, 7, 841 (2005).

13. Y.-H. Liu, Y. Chen and J.-H. Yi, West China J. Pharmaceut. Sci., 17, 295 (2002).

14. L. Zhou, Food Sci. Technol., 33, 152 (2008).

15. X.-Y. Nie, C. Shang, Y.-L. Wang, B. Zhang and H.-B. Qu, Chinese Trad. Patent Med., 27, 350 (2005).

16. H. Liu, M. Wan, Y. Hao, X.-Y. Zhu, X.-R. Ta and D.-Q. Zhang, J. Tianjin Univ. Trad. Chinese Med., 28, 142 (2005).

17. Y.-P. Xing, B.-C. Lin, W.-H. Zhou, X.-D. Tang and H. Gao, Fine Chem., 26, 666 (2009). 